[Article]

\title{
苯硫酚盐基溶液的可充镁电池电解液
}

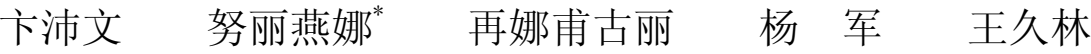 \\ (上海交通大学化学化工学院, 上海 200240)
}

\begin{abstract}
摘要：将4-甲基苯硫酚、4-异丙基苯硫酚和 4-甲氧基苯硫酚(RSH)分别与格氏试剂 $\mathrm{C}_{2} \mathrm{H}_{5} \mathrm{MgCl} / \mathrm{THF}$ (四氢呋喃) 反应制得的苯硫酚氯化镁 $\left(R S M g C l\right.$ ) (分别标记为 MBMC、IPBMC 和 MOBMC)/THF 和进一步与 Lewis 酸 $\mathrm{AICl}_{3}$ 反应制得的 $(\mathrm{RSMgCl})_{n}$ - $\mathrm{AICl}_{3} / \mathrm{THF}(n=1,1.5,2)$ 苯硫酚盐基溶液用作可充镁电池电解液, 采用循环伏安和恒电 流充放电测试研究了电解液的镁沉积-溶出性能和氧化分解电位. 结果表明, 苯硫酚上的基团种类和 $\mathrm{RSMgCl}$ 与 $\mathrm{AlCl}_{3}$ 的比例对其电化学性能有影响. 其中, $0.5 \mathrm{~mol} \cdot \mathrm{L}^{-1}$ (IPBMC) $)_{1.5}-\mathrm{AlCl}_{3} / \mathrm{THF}$ 溶液具有最佳的电化学性能, 其氧化分解电位适宜 $\left(2.4 \mathrm{~V}\left(\mathrm{vs} \mathrm{Mg} / \mathrm{Mg}^{2+}\right)\right)$, 镁沉积-溶出循环效率稳定, 过电位低, 电导率较高 $\left(2.48 \mathrm{mS} \cdot \mathrm{cm}^{-1}\right)$, 与正极材料 $\mathrm{Mo}_{6} \mathrm{~S}_{8}$ 兼容性良好, 且具有一定的空气稳定性, 配制方便, 有希望应用于实际的可充镁电池体系中.
\end{abstract}

关键词：可充镁电池；电解液；电化学性能；空气稳定性；兼容性

中图分类号: 0646

\section{Benzenethiolate-Based Solutions for Rechargeable Magnesium Battery Electrolytes}

\author{
BIAN Pei-Wen NULI Yan-Na* Zainapuguli YANG Jun WANG Jiu-Lin \\ (School of Chemical Engineering, Shanghai Jiao Tong University, Shanghai 200240, P. R. China)
}

\begin{abstract}
The benzenethiolate-based solutions ( $\mathrm{RSMgCl})_{n}-\mathrm{AlCl}_{3} /$ tetrahydrofuran (THF) (R=4-methylbenzene, 4-isopropylbenzene, 4-methoxybenzene; $n=1,1.5,2$, respectively) were obtained by the simple reaction of benzenethiol compounds with the Grignard reagent $\mathrm{C}_{2} \mathrm{H}_{5} \mathrm{MgCl} / \mathrm{THF}$ and $\mathrm{AlCl}_{3}$ in $\mathrm{THF}$, and the electrochemical performance as the rechargeable magnesium battery electrolytes are reported. First, 4-methyl-benzenethiolate magnesium chloride (MBMC)/THF, 4-isopropylbenzenethiolate magnesium chloride (IPBMC)/THF, and 4methoxybenzenethiolate magnesium chloride (MOBMC)/THF solutions (termed as RSMgCl/THF) were synthesized by the reaction of 4-methylbenzenethiol, 4-isopropylbenzenethiol, and 4-methoxybenzenethiol compounds, respectively, with $\mathrm{C}_{2} \mathrm{H}_{5} \mathrm{MgCl} / \mathrm{THF}$ via a hydrogen metal-radical exchange with rapid evolution of ethane gas. Furthermore, $(\mathrm{RSMgCl})_{n}-\mathrm{AlCl}_{3} / \mathrm{THF}$ solutions were obtained by the reaction of $\mathrm{RSMgCl} / \mathrm{THF}$ with $\mathrm{AlCl}_{3} / \mathrm{THF}$ at different molar ratios of $\mathrm{RSMgCl}: \mathrm{AlCl}_{3}$. The benzenethiolate-based solutions as electrolytes for rechargeable magnesium batteries were characterized in term of anodic stability and reversibility of magnesium deposition-dissolution using cyclic voltammetry and galvanostatic charge/discharge techniques. Furthermore, the compatibility of the solutions with $\mathrm{Mo}_{6} \mathrm{~S}_{8}$ cathode material was verified using coin cells with a $\mathrm{Mo}_{6} \mathrm{~S}_{8}$ cathode, $\mathrm{Mg}$ anode, and benzenethiolate-based electrolyte. It is concluded that both the substituents on benzenethiol and the ratio of $\mathrm{RSMgCl}: \mathrm{AlCl}_{3}$ have an effect on the electrochemical performance. $0.5 \mathrm{~mol} \cdot \mathrm{L}^{-1}(\mathrm{IPBMC})_{1.5}-\mathrm{AlCl}_{3} /$ THF shows the best electrochemical performance with $2.4 \mathrm{~V}\left(\mathrm{vs} \mathrm{Mg} / \mathrm{Mg}^{2+}\right)$ anodic stability, a low voltage for magnesium deposition-dissolution, a high cycling reversibility, and good compatibility with the $\mathrm{Mo}_{6} \mathrm{~S}_{8}$ cathode. Moreover, the air insensitive character and easy preparation make it a promising candidate for rechargeable battery electrolytes.
\end{abstract}

Received: September 24, 2013; Revised: December 20, 2013; Published on Web: December 20, 2013.

"Corresponding author. Email: nlyn@sjtu.edu.cn; Tel: +86-21-5474-5887-16.

The project was supported by the National Natural Science Foundation of China (21273147) and Shanghai Municipal Science and Technology Commission, China (11JC1405700).

国家自然科学基金(21273147)和上海市科委(11JC1405700)资助项目

(c) Editorial office of Acta Physico-Chimica Sinica 
Key Words: Rechargeable magnesium battery; Electrolyte; Electrochemical performance; Air insensitive character; Compatibility

\section{1 引言}

近年来, 能源、材料和信息已然成为人类社会 发展中的三大重要产业, 其中能源是实现整个世界 向前发展和经济快速增长最基本的动力. 在现有 的化学电源技术中, 锂离子电池因其能量密度大、 技术成熟而被广泛应用于各个领域, 但由于存在安 全性较低和成本较高的问题, 在大型电能储存设备 和电动汽车动力电池方面的应用受到一定限制. 人 们开始注意到在元素周期表上与锂处于对角线位 置的镁, 根据对角线规则, 二者具有较为相似的物 理和化学性能, 且镁储量十分丰富, 具有成本低、电 极电位较低、理论容量较高、加工处理方便、对环境 无污染等诸多优点, 因此以金属镁为负极的可充镁 电池被认为在大型电能储存设备和电动汽车动力 电池方面很有发展前景.

由于镁的反应活性较高, 在大多数电解液中都 会形成表面针化膜, 致使镁离子无法穿过, 从而难 以进行可逆的镁沉积和溶出, 限制了其电化学活 性. 研究表明, 在格氏试剂 $(\mathrm{RMgX}, \mathrm{R}$ 为烷基或芳基, $X$ 为卤素)、 ${ }^{1-4} \mathrm{Mg}\left(\mathrm{BR}_{2} \mathrm{R}_{2}^{\prime}\right)_{2}$ (其中 $R 、 R^{\prime}$ 为烷基或芳 基)、 ${ }^{5-7} \mathrm{Mg}\left(\mathrm{AX}_{4-n} \mathrm{R}_{n} \mathrm{R}_{n^{\prime}}^{\prime}\right)_{2}$ 络合物(其中 $\mathrm{A}=\mathrm{Al}, \mathrm{B}, \mathrm{Sb}, \mathrm{P}$, $\mathrm{As}, \mathrm{Fe}, \mathrm{Ta}$ 等; $\mathrm{X}=\mathrm{Cl}, \mathrm{Br}, \mathrm{F} ; \mathrm{R} 、 \mathrm{R}^{\prime}$ 为烷基或芳基, $0<n<$ $\left.4, n^{\prime}+n^{\prime \prime}=n\right)^{8-13}$ 和氨基镁卤化物 ${ }^{5-7}$ 等溶于醚形成的有 机溶液体系中, 可以实现镁的可逆沉积. 其中突破 性的进展是由 Aurbach等 ${ }^{8-13}$ 提出的 $\mathrm{Mg}\left(\mathrm{AlCl}_{3-n} \mathrm{R}_{n} \mathrm{R}^{\prime}\right)_{2}$ (其中 $\mathrm{R}$ 和 $\mathrm{R}^{\prime}$ 为烷基)的醚溶液体系, 在 $0.25 \mathrm{~mol} \cdot \mathrm{L}^{-1}$ $\mathrm{Mg}\left(\mathrm{AlCl}_{2} \mathrm{BuEt}\right)_{2} / \mathrm{THF}$ (其中 $\mathrm{Bu}$ 为丁基, $\mathrm{Et}$ 为乙基, THF 为四氢呋喃)的铝基电解液 (被称为 “一代” 电解 液, 该电解液由 Lewis 碱 $\mathrm{MgBu}_{2}$ 和 Lewis 酸 $\mathrm{AlEtCl}_{2}$ 按 1:2 摩尔比反应制得)中, 呈现出极高的镁沉积-溶 出的可逆性, 其氧化分解电位为 $2.5 \mathrm{~V}\left(v \mathrm{Mg} / \mathrm{Mg}^{2+}\right)$ (研究中发现电解液在不同电极上的氧化分解电 位差异较大, 不特别说明时表示的是对铂电极而 $\left.{ }^{(14}\right)$, 室温电导率为 $1-2 \mathrm{mS} \cdot \mathrm{cm}^{-1}$. 此体系主要的问 题是合成过程复杂, 所用溶剂 THF 挥发性较大, 使 电解液不宜长期放置, 氧化分解电位仍然较低. Aurbach 小组 ${ }^{15-17}$ 接着将烷基卤铝络合物中的烷基 基团全部换为苯基, 得到了全苯基镁铝卤络合物/ THF 的铝基电解液 (被称为 “二代” 电解液), 该电解 液由 Lewis 碱 $\mathrm{PhMgCl}$ (其中 $\mathrm{Ph}$ 为苯基)和 Lewis 酸
$\mathrm{AlCl}_{3}$ 按 2:1 摩尔比在 THF 中反应制得. 相比于 “一 代”电解液, 该电解液的氧化分解电位可达到 $3 \mathrm{~V}(v \mathrm{~s}$ $\mathrm{Mg} / \mathrm{Mg}^{2+}$ ), 电导率也显著提高, 镁离子能够较快、可 逆地插入到 $\mathrm{Mo}_{6} \mathrm{~S}_{8}$ 正极材料中. Muldoon 等 7,18 将氨 基镁氯化物 $\mathrm{HMDSMgCl}$ (其中 $\mathrm{HMDS}$ 为 $\left(\mathrm{Me}_{3} \mathrm{Si}\right)_{2} \mathrm{~N}$ ) 与 $\mathrm{AlCl}_{3}$ 反应改性后, 电解液的氧化分解电位可达 $3.2 \mathrm{~V}\left(\mathrm{vs} \mathrm{Mg} / \mathrm{Mg}^{2+}\right)$. 我们课题组 ${ }^{1920}{ }^{2}$ 提出的硼基电解 液 $\mathrm{Mes}_{3} \mathrm{~B}-(\mathrm{PhMgCl})_{2} / \mathrm{THF}$ (其中 $\mathrm{Mes}_{3} \mathrm{~B}$ 为三 3 , 5 -二甲 基苯基)嗍烷), 可将电解液的阳极稳定性提高到 3.5 $\mathrm{V}\left(v s \mathrm{Mg} / \mathrm{Mg}^{2+}\right)$, 但这些电解液均对湿气具有敏感 性, 不能暴露于空气中. 另外, 考虑到制备过程简单 易行、原材料价格便宜, 我们还利用格氏试剂与活 泼氢的反应, 分别制备并研究了吡咯烷基和苯酚盐 基电解液, 其氧化分解电位可分别达到 2.3 和 $2.6 \mathrm{~V}$ $\left(v s \mathrm{Mg} / \mathrm{Mg}^{2+}\right)$, 具有稳定的镁沉积-溶出性能; 2 ; , 22 并进 一步采用具有两个活泼氢的乙烯硫胒与不同类型 的格氏试剂相反应, 研究了格氏试剂类型对电解液 的氧化分解电位和镁的沉积-溶出效率、循环稳定性 能的影响. ${ }^{23}$ 本文将常见的格氏试剂与含有活泼氢 的三种苯硫酚反应, 并分别进一步与 $\mathrm{AlCl}_{3}$ 在不同 比例下混合生成具有一定空气稳定性的苯硫酚盐 基电解液, 研究了苯硫酚上的基团种类和 $\mathrm{RSMgCl}$ 与 $\mathrm{AlCl}_{3}$ 的摩尔比对其电化学性能的影响.

\section{2 实验部分}

\section{1 试剂与仪器}

4-甲基苯硫酚(98\%)、4-异丙基苯硫酚(95\%)、4甲氧基苯硫酚 $(97 \%)$ 购于上海百灵威化学技术有限 公司, $2 \mathrm{~mol} \cdot \mathrm{L}^{-1} \mathrm{CH}_{3} \mathrm{CH}_{2} \mathrm{MgCl} / \mathrm{THF}$ 格氏试剂和镁条 (纯度 $>99.99 \%$ ) 购于 Aldrich, 无水 $\mathrm{AlCl}_{3}$ (98\%) 购于 Alfa Aesar, 四氢呋喃使用之前经实验室自组装设备 重蒸纯化处理去除微量水. 所有试剂放置于氞气气 氛手套箱(MBRAUN, 德国 UNILAB) 中备用. 铂(Pt) 盘 $(d=2 \mathrm{~mm})$ 电极购于上海仙仁仪器仪表有限公司.

使用 CHI660 电化学工作站(上海辰华仪器有限 公司)作循环伏安扫描测试, LAND-CT2001A 系统 测试扣式电池中电解液的镁沉积与溶出循环特性. FE30 电导率仪和 inLab710 电极 (Mettler Toledo, Switzerland)测试电解液的电导率. D/max-2200/PC 型 $\mathrm{X}$ 射线衍射仪(XRD, 日本 Rigaku 公司)分析沉积 
在金属表面的组分.

\section{2 实验方法}

\subsection{1 电解液配置}

在氩气手套箱内, 室温条件下, 取 $2 \mathrm{mmol}$ 的 4甲基苯硫酚、4-异丙基苯硫酚、4-甲氧基苯硫酚分别 加入到干净的圆底烧瓶中, 加入 $1 \mathrm{~mL}$ 的 THF 搅拌 后, 再加入 $1 \mathrm{~mL}$ 的 $\mathrm{CH}_{3} \mathrm{CH}_{2} \mathrm{MgCl} / \mathrm{THF}$ 溶液(反应物 的摩尔比为 $1: 1$ )摚拌, 得到三种 $1 \mathrm{~mol} \cdot \mathrm{L}^{-1} \mathrm{RSMgCl} /$ $\mathrm{THF}$ 溶液, 其中 $\mathrm{RSMgCl}$ 分别命名为 MBMC (4methyl-benzenethiolate magnesium chloride, 4- 甲基 苯硫酚氯化镁), IPBMC (4-isopropylbenzenethiolate magnesium chloride, 4-异丙基苯硫酚氯化美), MOBMC (4-methoxybenzenethiolate magnesium chloride, 4-甲 氧基苯硫酚氯化镁). 反应式(1)为 4-甲基苯硫酚和 $\mathrm{CH}_{3} \mathrm{CH}_{2} \mathrm{MgCl}$ 的反应, 其它两种苯硫酚与 $\mathrm{CH}_{3} \mathrm{CH}_{2} \mathrm{MgCl}$ 反应与(1)式类似,

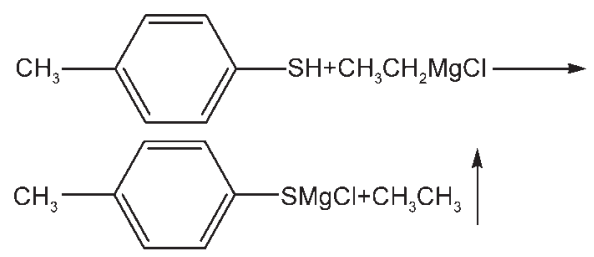

再将 $\mathrm{AlCl}_{3}$ 通过搅拌溶于 $\mathrm{THF}$ 中 (摩尔比为 $1: 2$ ), 分 别与 $\mathrm{RSMgCl} / \mathrm{THF}$ 在不同摩尔比下混合, 得到 0.5 $\mathrm{mol} \cdot \mathrm{L}^{-1}(\mathrm{RSMgCl})_{n}-\mathrm{AlCl}_{3} / \mathrm{THF}(n=1,1.5,2)$.

2.2.2 $\mathrm{Mo}_{6} \mathrm{~S}_{8}$ 正极材料的制备

首先通过熔盐法合成 Chevrel 相 $\mathrm{Cu}_{2} \mathrm{Mo}_{6} \mathrm{~S}_{8}$, 反应 如(2)式所示: ${ }^{24}$

$$
2 \mathrm{CuS} \cdot \mathrm{H}_{2} \mathrm{O}+3 \mathrm{MoS}_{2}+3 \mathrm{Mo} \rightarrow
$$$$
\mathrm{Cu}_{2} \mathrm{Mo}_{6} \mathrm{~S}_{8}+2 \mathrm{H}_{2} \mathrm{O} \uparrow
$$

按照化学计量比称取 $\mathrm{CuS} \cdot \mathrm{H}_{2} \mathrm{O} 、 \mathrm{MoS}_{2} 、 \mathrm{Mo}$ 粉末 以及熔盐 $\mathrm{KCl}$ (反应物与熔盐摩尔比为 $1: 2$ ), 研磨使 其均匀混合, 在氩气气氛保护下于 $850{ }^{\circ} \mathrm{C}$ 的温度下 烧结 $60 \mathrm{~h}$, 升温速率为 $2{ }^{\circ} \mathrm{C} \cdot \mathrm{min}^{-1}$, 然后自然冷却至 室温. 所得粉末用热的去离子水超声清洗 3-4 次, 离 心后 $110{ }^{\circ} \mathrm{C}$ 下真空干燥 $2 \mathrm{~h}$, 得到 $\mathrm{Cu}_{2} \mathrm{Mo}_{6} \mathrm{~S}_{8}$.

将 $1.2 \mathrm{~g}$ 的 $\mathrm{Cu}_{2} \mathrm{Mo}_{6} \mathrm{~S}_{8}$ 材料放入 $20 \mathrm{~mL}$ 的盐酸水 溶液 $\left(\mathrm{HCl}: \mathrm{H}_{2} \mathrm{O}\right.$ 体积比为 1:1) 中, 在室温下搅拌 2-4 天, 过程中通 $7 \mathrm{~h} \mathrm{O}_{2}\left(100 \mathrm{~cm}^{3} \cdot \mathrm{min}^{-1}\right)$ 以加速反应进 行, 其反应方程式如式(3)所示. ${ }^{25}$ 之后将该溶液离 心, 用 $100 \mathrm{~mL}$ 去离子水清洗3-4 次, 离心后 $120^{\circ} \mathrm{C}$ 下真空干燥 $2 \mathrm{~h}$, 得到 $\mathrm{Mo}_{6} \mathrm{~S}_{8}$ 正极材料.

$$
\begin{aligned}
& \mathrm{Cu}_{2} \mathrm{Mo}_{6} \mathrm{~S}_{8}(\mathrm{~s})+8 \mathrm{HCl}(\mathrm{aq})+\mathrm{O}_{2} \rightarrow \\
& \mathrm{Mo}_{6} \mathrm{~S}_{8}(\mathrm{~s})+2 \mathrm{H}_{2} \mathrm{O}+2\left[\mathrm{CuCl}_{4}\right]^{2-}(\mathrm{aq})+4 \mathrm{H}^{+}(\mathrm{aq})
\end{aligned}
$$

\subsection{3 电化学性能的测试}

电解液中镁的电化学沉积-溶出性能通过循环 伏安 $(\mathrm{CV})$ 曲线来测定, 测试过程均在室温、氩气手 套箱中进行. 实验采用三电极体系, 工作电极为铂 盘电极, 使用前用三氧化铝粉末进行抛光处理, 然 后用去离子水及无水乙醇清洗, 烘干后使用. 参比 电极、对电极均采用表面用 $800 \mathrm{Cw}$ 砂纸打磨处理干 净的镁条. 测试用的三电极管及塞子均经过烘干处 理. 测量时, 从开路电位开始向负方向扫描, 扫描速 率为 $50 \mathrm{mV} \cdot \mathrm{s}^{-1}$.

通过 CR2016 扣式电池的恒电流充放电测试电 解液中镁的电化学沉积-溶出循环性能. 扣式电池的 组装在氩气气氛手套箱中进行. 采用的正极为铜䈃 $(99.99 \%$ )、负极为镁条(使用前用 $800 \mathrm{Cw}$ 砂纸打磨光 亮), 隔膜为 Entek PE 膜; 电池组装后在室温放置 $4 \mathrm{~h}$ 后进行测量. 充放电过程中的电流密度为 $0.1 \mathrm{~mA}$. $\mathrm{cm}^{-2}$, 镁的沉积过程通过放电时间控制, 而镁的脱出 通过充电极限电位到 $0.8 \mathrm{~V}$ 来控制, 每次充电与放电 过程之间缓冲时间为 $30 \mathrm{~s}$.

按质量比 8:1:1 称取正极活性材料 $\mathrm{Mo}_{6} \mathrm{~S}_{8}$ 、 Super-P 导电碳和聚偏氟乙烯(PVDF)粘结剂(PVDF 预先溶于 $N$-甲基吡咯烷酮 (NMP) 中配成浓度为 $0.02 \mathrm{~g} \cdot \mathrm{mL}^{-1}$ 的溶液)置于小烧杯中, 磁力搅拌 $4 \mathrm{~h}$ 得 到混合均匀的粘稠浆料. 将浆料均匀涂敷于 $\mathrm{Cu}$ 簿集 流体上, 冲成均一的极片, 并在 $120^{\circ} \mathrm{C}$ 下真空干燥 5 $\mathrm{h}$ 后, 迅速转移至氩气手套箱中, 以打磨光亮的镁片 为负极, Entek PE膜为隔膜, 与上面自制的电解液一 起组装成 CR2016 扣式可充镁电池. 组装好的电池 在室温下静置 $4 \mathrm{~h}$, 使电解液完全浸润后进行充放电 性能测试.

\subsubsection{XRD 测试}

恒电流条件下进行镁在金属 $\mathrm{Cu}$ 基质上的沉积, 沉积电量为 $85.68 \mathrm{C} \cdot \mathrm{cm}^{-2}$. 实验采用两电极体系, 室 温条件下在氩气气氛的手套箱中进行测定, 仪器设 备采用 CHI660C 电化学工作站; 正极为铜片, 表面 用无水乙醇擦拭干净; 负极为镁条, 使用前用 $800 \mathrm{Cw}$ 砂纸打磨光亮.

将沉积好的扣式电池在氩气气氛手套箱中打 开, $\mathrm{Cu}$ 片上的沉积样品用重蒸后的 THF 溶剂进行冲 洗, 晾干后密封取出, 使用 $\mathrm{X}$ 射线衍射仪 $(\mathrm{Cu}$ 靶)对铜 基质上的电沉积物进行 XRD 检测, 扫描范围为 $30^{\circ}-75^{\circ}$, 扫描速率为 $4.0\left(^{\circ}\right) \cdot \mathrm{min}^{-1}$. 


\section{3 结果与讨论}

\section{$3.1 \mathrm{RSMgCl} / \mathrm{THF}$ 电解液}

图 1 为 4-甲基苯硫酚、4-异丙基苯硫酚、4-甲氧 基苯硫酚与 $\mathrm{CH}_{3} \mathrm{CH}_{2} \mathrm{MgCl} / \mathrm{THF}$ 格氏试剂以 $1: 1$ 摩尔 比反应配制的 $1 \mathrm{~mol} \cdot \mathrm{L}^{-1} \mathrm{RSMgCl}(\mathrm{MBMC} 、 \mathrm{IPBMC}$ 和 MOBMC)/THF 溶液在铂盘电极上的循环伏安曲 线. 三种溶液中都可以进行镁的电化学沉积和溶 出, 且沉积-溶出曲线差别不大. 在从开路电位向负 向扫描的过程中, 从 $-0.2 \mathrm{~V}\left(v \mathrm{sg} / \mathrm{Mg}^{2+}\right)$ 以下开始出 现沉积电流, 沉积过程中呈现一个典型的由过电位 驱动产生晶核及晶核生长的成核环; 正向扫描时, 从 $0 \mathrm{~V}\left(v s \mathrm{Mg} / \mathrm{Mg}^{2+}\right)$ 左右电流开始上升, 表示镁开始 溶出, 在 $0.7 \mathrm{~V}\left(v s \mathrm{Mg} / \mathrm{Mg}^{2+}\right)$ 附近出现的峰为美的氧 化溶出峰; 然后, 电流值趋于稳定, 基本不变, 表明 镁溶出过程结束; 继续向正方向扫描, 重新开始出 现电流上升, 此时的过程为电解液的氧化分解, 刚 开始出现电流明显上升所对应的电位为电解液的 氧化分解电位. 从图中可以得出 $\mathrm{MBMC} / \mathrm{THF}$ 、 IPBMC/THF、MOBMC/THF 溶液的氧化分解电位分 别为 $1.55 、 1.65$ 和 $1.85 \mathrm{~V}\left(v s \mathrm{Mg} / \mathrm{Mg}^{2+}\right)$. 硫酚中苯环 上取代基的类型对电解液的氧化分解电位有明显 影响. 在苯环相同的对位上引入供电子基团, 离域 的电子可与苯环形成稳定的共轭结构, 从而稳定

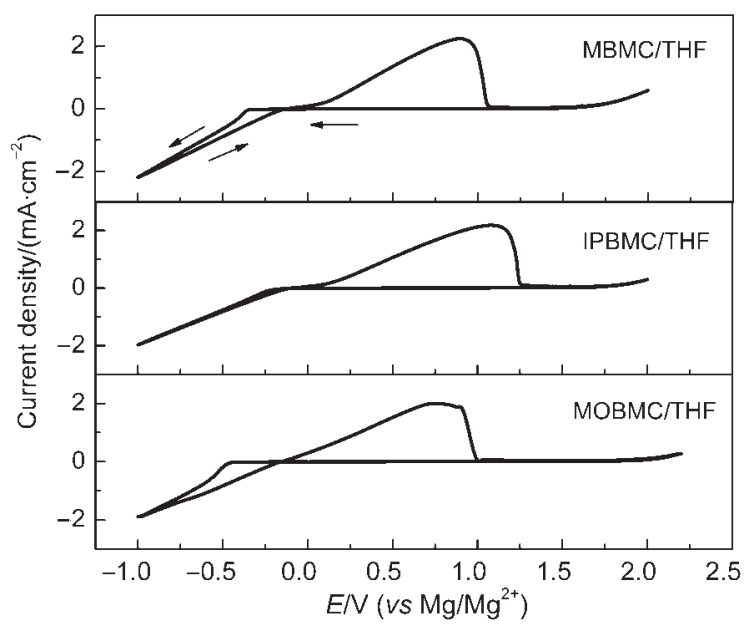

图 $11 \mathrm{~mol} \cdot \mathrm{L}^{-1}$ 的 MBMC/THF、IPBMC/THF、MOBMC/ THF 电解液中镁在铂盘电极上沉积-溶出的 典型循环伏安曲线

Fig.1 Typical cyclic voltammetry (CV) curves of Mg deposition-dissolution on Pt disk electrode in $1 \mathrm{~mol} \cdot \mathrm{L}^{-1}$ MBMC/THF, IPBMC/THF, and MOBMC/THF electrolytes MBMC: 4-methyl-benzenethiolate magnesium chloride; IPBMC:

4-isopropylbenzenethiolate magnesium chloride; MOBMC: 4methoxybenzenethiolate magnesium chloride; THF: tetrahydrofuran
$\mathrm{R}-\mathrm{Mg}$ 键, 提高其电化学稳定性. 文中采用了供电 子能力差异明显的三种基团, 其中, 甲氧基供电子 能力最强, 其次为异丙基, 最后为甲基, 因此电解液 的氧化分解电位顺序为 MOBMC/THF $>$ IPBMC/ THF $>$ MBMC/THF.

\section{$3.2(\mathrm{RSMgCl})_{2}-\mathrm{AlCl}_{3} / \mathrm{THF}$ 电解液}

鉴于 “二代”电解液由 Lewis 碱 $\mathrm{PhMgCl}$ 和 Lewis 酸 $\mathrm{AlCl}_{3}$ 反应的制备思路, 我们接着将以上三种 $\mathrm{RSMgCl} / \mathrm{THF}$ 溶液分别与 $\mathrm{AlCl}_{3} / \mathrm{THF}$ 以 $2: 1$ 摩尔比 配制成 $(\mathrm{RSMgCl})_{2}-\mathrm{AlCl}_{3}$ 电解液, 比较了其中的美 沉积-溶出性能. 图 2 为三种 $0.5 \mathrm{~mol} \cdot \mathrm{L}^{-1}(\mathrm{RSMgCl})_{2}-$ $\mathrm{AlCl}_{3} / \mathrm{THF}$ 电解液在铂盘电极上的循环伏安曲线. 可以看出, 加入 $\mathrm{AlCl}_{3} / \mathrm{THF}$ 后, 电解液仍具有镁的沉 积-溶出性能, 且电解液体系沉积-溶出电流值变大, 阳极氧化分解电位有了很明显的改善, 分别从 1.55 、 1.65 和 $1.85 \mathrm{~V}$ 上升到了 2.3、2.4 和 $2.2 \mathrm{~V}$. 其中, (IPBMC) $)_{2}-\mathrm{AlCl}_{3} / \mathrm{THF}$ 氧化分解电位与 “一代”电解液 相当, 且具有优异的镁沉积 - 溶出性能, 下文对 IPBMC 与 $\mathrm{AlCl}_{3}$ 形成的电解液体系进行更深入的研 究.

\section{3 (IPBMC $)_{n}-\mathrm{AlCl}_{3} / \mathrm{THF}(n=1,1.5,2)$ 电解液}

Aurbach 小组 ${ }^{10}$ 在对铝基电解液的研究过程中 发现, 不同路易斯酸碱的摩尔比对电解液的电化学 性能有显著的影响. 基于此考虑, 我们将上面得到 的 IPBMC 路易斯碱与 $\mathrm{AlCl}_{3}$ 路易斯酸以不同摩尔比

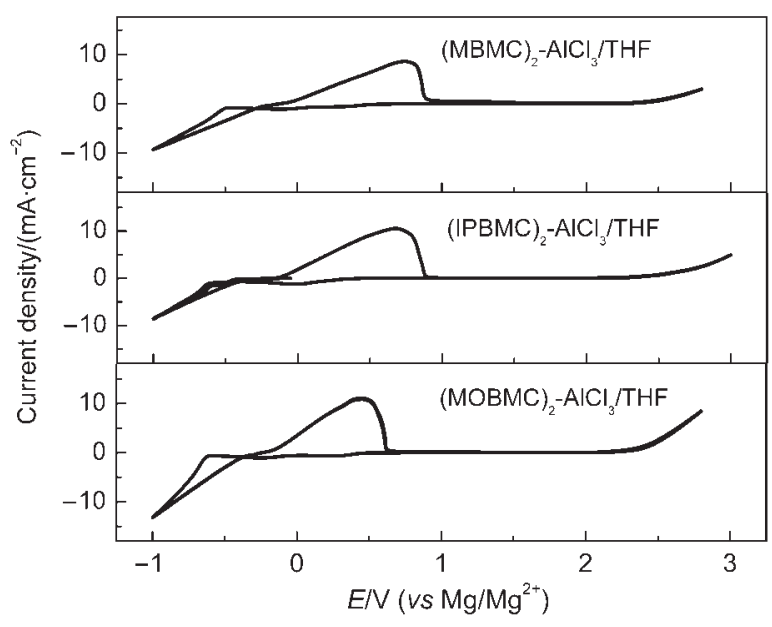

图 $20.5 \mathrm{~mol} \cdot \mathrm{L}^{-1}\left(\mathrm{MBMC}_{2}-\mathrm{AlCl}_{3} / \mathrm{THF}\right.$ ( $(\mathrm{IPBMC})_{2}-\mathrm{AlCl}_{3} /$ THF、(MOBMC $)_{2}-\mathrm{AlCl}_{3} / \mathrm{THF}$ 电解液中镁在铂盘电极上 沉积-溶出的典型循环伏安曲线

Fig.2 Typical $\mathrm{CV}$ curves of $\mathrm{Mg}$ deposition-dissolution on Pt disk electrode in $0.5 \mathrm{~mol} \cdot \mathrm{L}^{-1}(\mathrm{MBMC})_{2}-\mathrm{AlCl}_{3} / \mathrm{THF}$, (IPBMC) $)_{2}-\mathrm{AlCl}_{3} / \mathrm{THF}$, and (MOBMC) $)_{2}-\mathrm{AlCl}_{3} / \mathrm{THF}$ electrolytes 
混合, 比较了其中的镁沉积-溶出性能. 图 3 为三种 $(\mathrm{IPBMC})_{n}-\mathrm{AlCl}_{3} / \mathrm{THF}(n=1,1.5,2)$ 电解液中镁沉积溶出的循环伏安性能. 图中显示, 不同比例电解液 的氧化分解电位相差不大, 均为 $2.4 \mathrm{~V}$ 左右, 但电流 密度随着比例的上升而下降. 电导率测试表明三种 溶液的电导率值分别为 $2.98 、 2.48$ 和 $1.57 \mathrm{mS} \cdot \mathrm{cm}^{-1}$, 与图 3 中的镁沉积-溶出的电流密度大小相符. 当 IPBMC 与 $\mathrm{AlCl}_{3}$ 摩尔比为 $1: 1$ 时, 氧化还原电流密度 值是最高的, 但该电解液放置一段时间后会出现沉 淀, 因而(IPBMC) $)_{1.5}-\mathrm{AlCl}_{3} / \mathrm{THF}$ 电解液为较佳的选 择.

\section{4 (IPBMC) $)_{1.5}-\mathrm{AlCl}_{3} / \mathrm{THF}$ 电解液}

鉴于现开发的可充镁电池电解液体系多在空 气中不稳定, 接触空气后就不再能够进行镁的可逆 沉积和溶出, 电化学活性丧失. 因此, 我们进一步考 察了苯硫酚盐基电解液体系在空气中的稳定性. 图 4 为将 $0.5 \mathrm{~mol} \cdot \mathrm{L}^{-1}(\mathrm{IPBMC})_{1}-\mathrm{AlCl}_{3} / \mathrm{THF}$ 电解液暴露 在空气中 $1 \mathrm{~h}$ 后的循环伏安曲线. 可以看出, 镁仍能 够在该电解液中进行可逆的沉积和溶出, 且氧化分 解电位与在真空操作条件下的差别不大, 镁的沉积溶出电流稍有降低(溶出峰电流密度从 $17 \mathrm{~mA} \cdot \mathrm{cm}^{-2}$ 降为 $12 \mathrm{~mA} \cdot \mathrm{cm}^{-2}$ ), 说明该电解液体系对空气中的 氧气和水分敏感性不强烈, 在空气中具有一定的稳 定性. 但实验中将电解液暴露在空气中超过 $1 \mathrm{~h}$ 后,

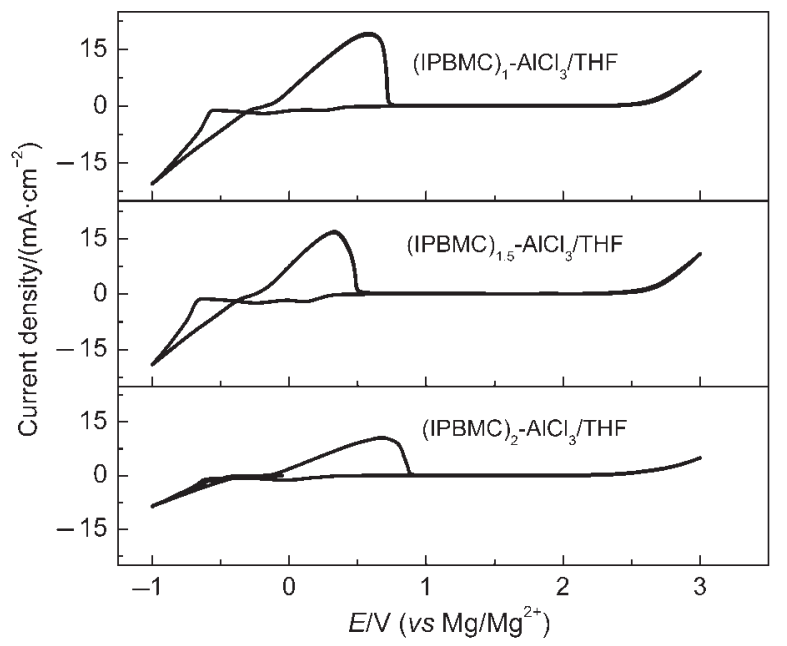

图 $30.5 \mathrm{~mol} \cdot \mathrm{L}^{-1}(\text { IPBMC) })_{1}-\mathrm{AlCl}_{3} / \mathrm{THF}$ (IPBMC) $)_{1.5}-\mathrm{AlCl}_{3} /$ THF、(IPBMC) $)_{2}-\mathrm{AlCl}_{3} / \mathrm{THF}$ 电解液中镁在铂盘电极上 沉积-溶出的典型循环伏安曲线

Fig.3 Typical $\mathrm{CV}$ curves of $\mathrm{Mg}$ deposition-dissolution on Pt disk electrode in $0.5 \mathrm{~mol} \cdot \mathrm{L}^{-1}(\mathrm{IPBMC})_{1}-\mathrm{AlCl}_{3} / \mathrm{THF}$, (IPBMC) $)_{1.5}-\mathrm{AlCl}_{3} / \mathrm{THF}$, and (IPBMC) $)_{2}-\mathrm{AlCl}_{3} / \mathrm{THF}$ electrolytes

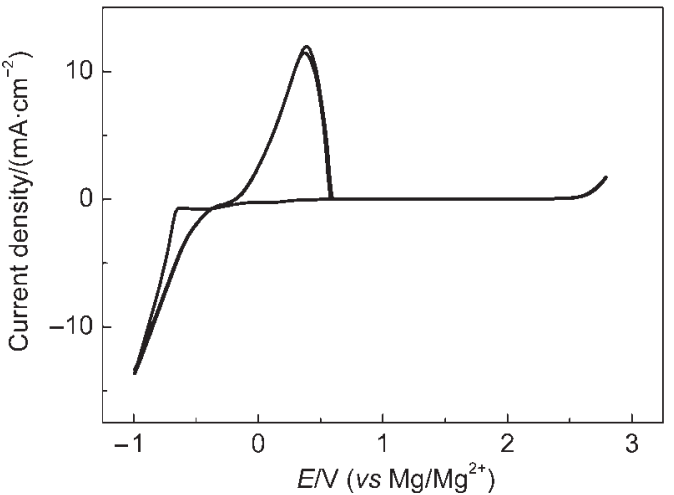

图 $40.5 \mathrm{~mol} \cdot \mathrm{L}^{-1}(\mathrm{IPBMC})_{1.5}-\mathrm{AlCl}_{3} / \mathrm{THF}$ 电解液在空气中 放置 $1 \mathrm{~h}$ 后在铂盘电极上的循环伏安曲线

Fig.4 CV curves of $\mathrm{Pt}$ disk electrode in $0.5 \mathrm{~mol} \cdot \mathrm{L}^{-1}$ (IPBMC) $)_{1.5}-\mathrm{AlCl}_{3} / \mathrm{THF}$ electrolyte exposed to air for $1 \mathrm{~h}$

电解液不再有电化学活性. 电解液在空气中的稳定 性有利于电解液制备过程的简化以及实际应用中 电池的组装.

图 5 为在 $0.5 \mathrm{~mol} \cdot \mathrm{L}^{-1}(\mathrm{IPBMC})_{1.5}-\mathrm{AlCl}_{3} / \mathrm{THF}$ 电 解液中, 以 $85.68 \mathrm{C} \cdot \mathrm{cm}^{-2}$ 电量在 $\mathrm{Cu}$ 基底表面沉积所 得到沉积物的 XRD 图谱. 图中在 $32.2^{\circ} 、 34.4^{\circ} 、 36.6^{\circ}$ 、 $47.8^{\circ} 、 57.4^{\circ} 、 63.1^{\circ} 、 68.7^{\circ}$ 和 $70.0^{\circ}$ 处的衍射峰为金属 镁的特征峰(JCPDS 35-0821), 43.3、50.4和 $74.1^{\circ}$ 为 铜基底的衍射峰, 除此之外没有别的峰, 这说明了 铜基底上的沉积物确实是金属镁.

镁电极在电解液体系中可逆沉积-溶出的过程 是表征可充镁电池电解液电化学性能的一个重要 参数, 可以通过扣式电池的恒电流循环充放电方法 测试. 图 6 显示了 $0.5 \mathrm{~mol} \cdot \mathrm{L}^{-1}(\mathrm{IPBMC})_{1.5}-\mathrm{AlCl}_{3} / \mathrm{THF}$ 溶液中 $\mathrm{Cu}$ 基底上镁的沉积-溶出循环曲线和效率

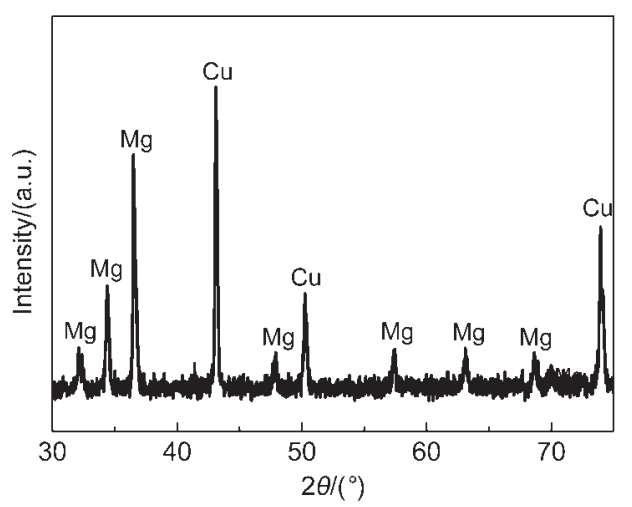

图 $50.5 \mathrm{~mol} \cdot \mathrm{L}^{-1}(\mathrm{IPBMC})_{1.5}-\mathrm{AlCl}_{3} / \mathrm{THF}$ 电解液体系中在 $\mathrm{Cu}$ 基底上沉积物的 $\mathrm{X}$ 射线衍射(XRD)谱图

Fig.5 X-ray diffraction (XRD) pattern of the electrodeposited compound on $\mathrm{Cu}$ substrate in $0.5 \mathrm{~mol} \cdot \mathrm{L}^{-1}$ (IPBMC) $)_{1.5}-\mathrm{AlCl}_{3} / \mathrm{THF}$ electrolyte 

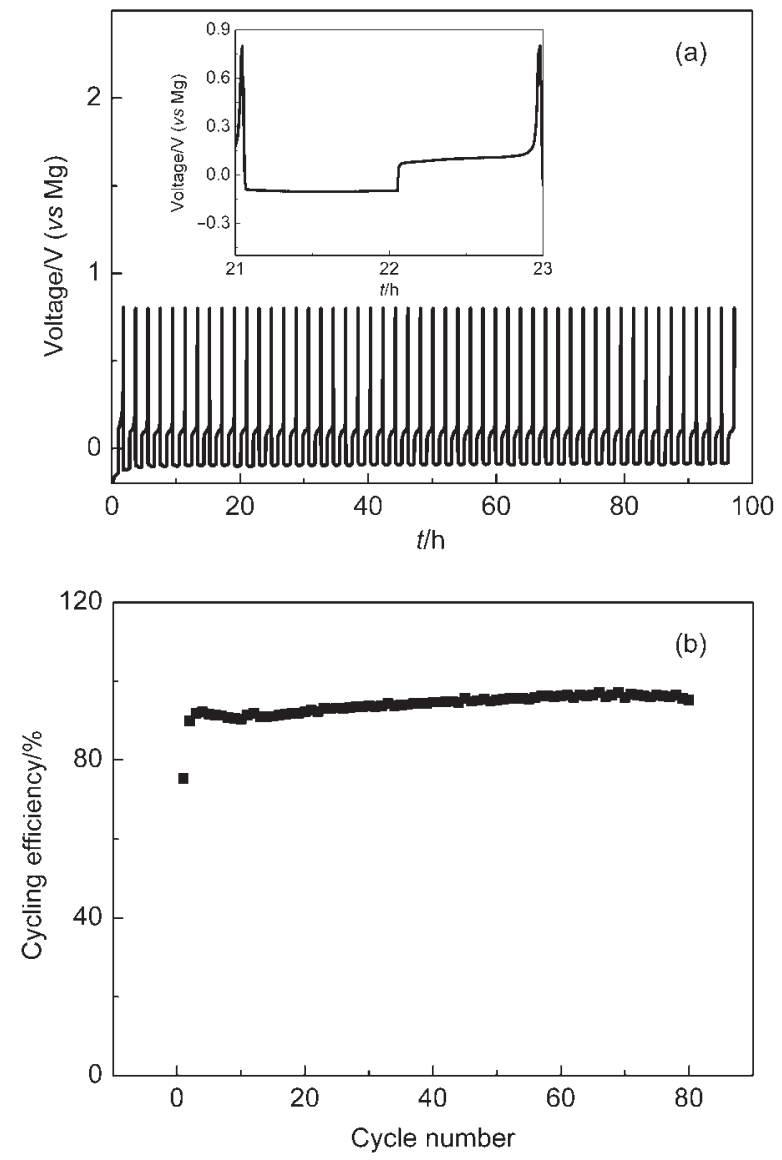

图 $60.5 \mathrm{~mol} \cdot \mathrm{L}^{-1}(\mathrm{IPBMC})_{1.5}-\mathrm{AlCl}_{3} / \mathrm{THF}$ 电解液中 $\mathrm{Cu}$ 基底上 的镁沉积-溶出循环曲线(a)和循环效率(b)

Fig.6 Mg deposition-dissolution cycling curves (a) and efficiency (b) on $\mathrm{Cu}$ substrate in $0.5 \mathrm{~mol} \mathrm{~L}^{-1}$ (IPBMC) $)_{1.5}-\mathrm{AlCl}_{3} / \mathrm{THF}$ electrolyte

Inset in the figure (a) is a typical galvanostatic curve.

(镁溶出的电量和镁沉积的电量之比). 由图中可以 看出稳定循环过程中美的沉积、溶出电位约为 \pm 0.1 $\mathrm{V}$, 镁在 $\mathrm{Cu}$ 基底上的首次沉积-溶出效率为 $75.4 \%$, 从第二个循环开始接近 $90 \%$, 循环稳定后效率可达 到 98.9\%. 说明该电解液具有较高的镁可逆沉积-溶 出循环效率.

为了证明所开发的苯硫酚盐基电解液体系可 以用于可充镁电池, 我们测试了(IPBMC) $)_{1.5}-\mathrm{AlCl}_{3} /$ THF 电解液与 Chevrel 相正极材料 $\mathrm{Mo}_{6} \mathrm{~S}_{8}$ 的兼容性. 采用 Chevrel 相的 $\mathrm{Mo}_{6} \mathrm{~S}_{8}$ 作为正极, 打磨光亮的镁片 作为负极, $0.5 \mathrm{~mol} \cdot \mathrm{L}^{-1}(\mathrm{IPBMC})_{1.5}-\mathrm{AlCl}_{3} / \mathrm{THF}$ 溶液作 为电解液组装成 CR2016 型扣式电池, 并对其进行 充放电性能测试. 测试条件采用 $0.37 C(1 C=122$ $\mathrm{mA} \cdot \mathrm{g}^{-1}$ ) 倍率, 充放电截止电压分别设为 1.7 和 0.5 $\mathrm{V}$, 结果如图 7 所示. 从图中可以看出, 该电池在较 大倍率 $(0.37 C)$ 下能够很好地进行充放电循环, 放电

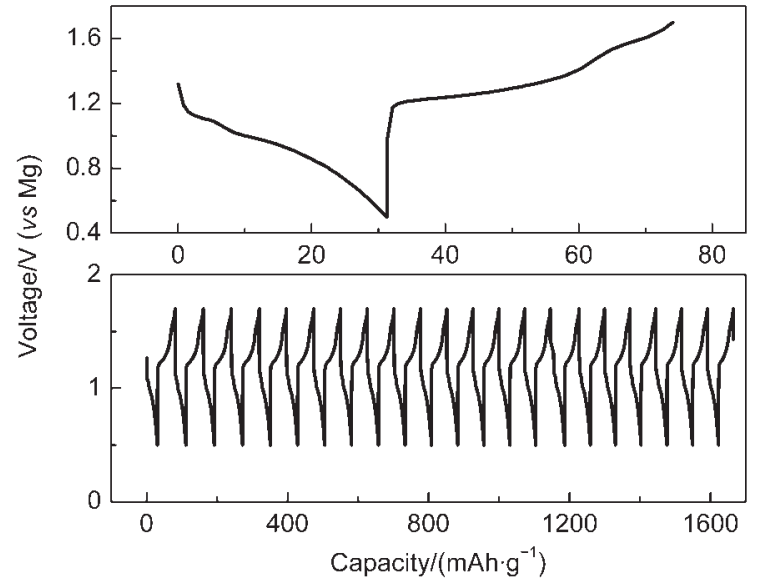

图 7 室温下 $0.5 \mathrm{~mol} \cdot \mathrm{L}^{-1}(\mathrm{IPBMC})_{1.5}-\mathrm{AlCl}_{3} / \mathrm{THF}$ 电解液中 $\mathrm{Mg} / \mathrm{Mo}_{6} \mathrm{~S}_{8}$ 扣式可充镁电池在 $0.37 \mathrm{C}$ 倍率下的充放电曲线

Fig.7 Charging and discharging curves of the rechargeable $\mathrm{Mg} / \mathrm{Mo}_{6} \mathrm{~S}_{8}$ coin-cell in $0.5 \mathrm{~mol} \mathrm{~L}^{-1}(\mathrm{PBMC})_{1.5^{-}}$ $\mathrm{AlCl}_{3} / \mathrm{THF}$ electrolyte at a current rate of $0.37 \mathrm{C}$ at room temperature

曲线表现出两个特征电压平台, 分别为 1.1 和 0.9 $\mathrm{V}$, 平均放电容量约为 $30 \mathrm{mAh} \cdot \mathrm{g}^{-1}$. 这些结果证明苯 硫酚盐基电解液与正极材料具有较好的兼容性, 有 望应用于实际的可充镁电池体系中.

\section{4 结 论}

本文通过简单的方法制备了一类含苯硫酚盐 的 THF 溶液作为可充镁电池的新型电解液, 运用循 环伏安、充放电测试对该类电解液中镁可逆沉积-溶 出性能和阳极氧化分解电位进行了系统的测试, 研 究表明该电解液体系具有较高的电导率、较高的阳 极氧化分解电位、可逆的镁沉积-溶出过程, 并具有 一定的空气稳定性, 与常用的可充镁电池正极材料 $\mathrm{Mo}_{6} \mathrm{~S}_{8}$ 具有好的兼容性, 能够应用于可充镁电池中.

\section{References}

(1) Genders, J. D.; Pletcher, D. J. Electroanal. Chem. Interfa. Electrochem. 1986, 199, 93. doi: 10.1016/0022-0728(86)87044-9

(2) Liebenow, C. J. J. Appl. Electrochem. 1997, 27, 221. doi: 10.1023/A:1018464210084

(3) Lu, Z.; Schechter, A.; Moshkovich, M.; Aurbach, D. J. Electroanal. Chem. 1999, 466, 203. doi: 10.1016/S0022-0728 (99)00146-1

(4) Aurbach, D.; Moshkovich, M.; Schechter, A.; Turgeman, R. Electrochem. Solid-State Lett. 2000, 3, 31.

(5) Gregory, T. D.; Hoffman, R. J.; Winterton, R. C. J. Electrochem. Soc. 1990, 137, 775. doi: 10.1149/1.2086553

(6) Liebenow, C.; Yang, Z.; Lobitz, P. Electrochem. Commun. 2000, 
2, 641. doi: 10.1016/S1388-2481(00)00094-1

(7) Muldoon, J.; Bucur, C. B.; Oliver, A. G.; Sugimoto, T.; Matsui, M.; Kim, H. S.; Allred, G. D.; Zajicek, J.; Kotani, Y. Energy Environ. Sci. 2012, 5, 5941. doi: 10.1039/c2ee03029b

(8) Aurbach, D.; Lu, Z.; Schechter, A.; Gofer, Y.; Gizbar, H.; Turgeman, R.; Cohen, Y.; Moshkovich, M.; Levi, E. Nature 2000, 407, 724. doi: 10.1038/35037553

(9) Aurbach, D.; Schechter, A.; Moshkovich, M.; Cohen, Y. J. Electrochem. Soc. 2001, 148, A1004.

(10) Aurbach, D.; Gizbar, H.; Schechter, A.; Chusid, O.; Gottlieb, H. E.; Gofer, Y.; Goldberg, I. J. Electrochem. Soc. 2002, 149, A115.

(11) Gizbar, H.; Vestfrid, Y.; Chusid, O.; Gofer, Y.; Gottlieb, H. E.; Marks, V.; Aurbach, D. Organometallics 2004, 23, 3826. doi: 10.1021/om049949a

(12) Vestfried, Y.; Chusid, O.; Gofer, Y.; Aped, P.; Aurbach, D. Organometallics 2007, 26, 3130. doi: 10.1021/om061076s

(13) Viestfried, Y.; Levi, M. D.; Gofer, Y.; Aurbach, D. J. Electroanal. Chem. 2005, 576, 183. doi: 10.1016/j. jelechem.2004.09.034

(14) Chen, Q.; Nuli, Y. N.; Yang, J.; Kailibinuer, K.; Wang, J. L. Acta Phys. -Chim. Sin. 2012, 28, 2625. [陈 强, 努丽燕娜, 杨 军, 凯丽比努尔·克日木, 王久林. 物理化学学报, 2012, 28, 2625.] doi: 10.3866/PKU.WHXB201208032

(15) Aurbach, D.; Suresh, G. S.; Levi, E.; Mitelman, A.; Mizrahi, O.; Chusid, O.; Brunelli, M. Adv. Mater. 2007, 19, 4260.

(16) Mizrahi, O.; Amir, N.; Pollak, E.; Chusid, O.; Marks, V.;
Gottlieb, H.; Larush, L.; Zinigrad, E.; Aurbach, D. J. Electrochem. Soc. 2008, 155, A103.

(17) Pour, N.; Gofer, Y.; Major, D. T.; Aurbach, D. J. Am. Chem. Soc. 2011, 133, 6270. doi: 10.1021/ja1098512

(18) Kim, H. S.; Arthur, T. S.; Allred, G. D.; Zajicek, J.; Newman, J. G.; Rodnyansky, A. E.; Oliver, A. G.; Boggess, W. C.; Muldoon, J. Nat. Commun. 2011, 2, 427. doi: 10.1038/ncomms 1435

(19) Guo, Y. S.; Zhang, F.; Yang, J.; Wang, F. F. Electrochem. Commun. 2012, 18, 24. doi: 10.1016/j.elecom.2012.01.026

(20) Guo, Y. S.; Zhang, F.; Yang, J.; Wang, F. F.; Nuli, Y. N.; Hirano, S. I. Energy Environ. Sci. 2012, 5, 9100. doi: 10.1039/ c2ee22509c

(21) Zhao, Q. S.; Nuli, Y. N.; Guo, Y. S.; Yang, J.; Wang, J. L. Electrochim. Acta 2011, 56, 6530. doi: 10.1016/j. electacta.2011.04.114

(22) Wang, F. F.; Guo, Y. S.; Yang, J.; Nuli, Y. N.; Hirano, S. I. Chem. Commun. 2012, 48, 10763. doi: 10.1039/c2cc35857c

(23) Bian, P. W.; Nuli, Y. N.; Chen, Q.; Yang, J.; Wang, J. L. Electrochemistry 2014, 20, 1. [市沛文, 努丽燕娜, 陈 强, 杨 军, 王久林. 电化学, 2014, 20, 1.]

(24) Lancry, E.; Levi, E.; Mitelman, A.; Malovany, S.; Aurbach, D. J. Solid State Chem. 2006, 179, 1879. doi: 10.1016/j. jssc.2006.02.032

(25) Lancry, E.; Levi, E.; Gofer, Y.; Levi, M.; Salitra, G.; Aurbach, D. Chem. Mater. 2004, 16, 2832. doi: 10.1021/cm034944+ 